\title{
Prediction Intervals based on Doubly Type-II Censored Data from Gompertz Distribution in the Presence of Outliers
}

\author{
S. F. Niazi Alil ${ }^{1}$ \\ Mathematics Department, \\ Faculty of Science and Human Studies \\ of Hotat Sudair, Majmaah University, \\ Majmaah 11952, Saudi Arabia. \\ Mathematics Department, \\ Faculty of Science, Al-Azhar University, \\ Assuit branch, 71524 Assiut, Egypt.
}

\author{
Ayed R. A. Alanzi ${ }^{2}$ \\ Mathematics Department, \\ Faculty of Science and Human Studies \\ of Hotat Sudair, Majmaah University, \\ Majmaah 11952, Saudi Arabia.
}

\begin{abstract}
The study aims at getting the Bayesian predication intervals for some order statistics of future observations from the distribution of Gompertz (Gomp $(\alpha, \beta)$ ). Doubly Type-II censored data has assisted obtaining in the presence of single outlier that arose from the different same family members of distribution. Single outlier of type $\beta \beta_{0}$ and $\beta+\beta_{0}$ are considered and bivariate independent prior density for $\alpha$ and $\beta$ are used. The problem of solving the Double integral to obtain the closed form for $\alpha$ and $\beta$, leads us to use MCMC for calculating the Bayesian Predication Intervals. The use of numerical examples and statistical data has enable to properly present and describe the procedure. We conclude that the Bayesian predication intervals are shorter for $y_{1}$ than $y_{5}$ when we are increasing the $\beta_{0}$ value.
\end{abstract}

Keywords-Bayesian prediction; Gompertz distribution; predictive distribution; doubly Type-II censored data; Markov Chain Monte Carlo; single outliers

\section{INTRODUCTION}

The adult death patterns can be effectively described through the use of the Gompertz distribution ([17]; [6]). Moreover, the Gompertz mortality force for the decreased infant and young adult levels of mortality extends to the whole life population span without any observed deceleration of mortality ([16]). A continuous probability density function (pdf) and a cumulative distribution function (cdf) are the constituents of the Gompertz distribution.

The $p d f$ as follows:

$$
f(x)=\alpha \beta e^{\alpha x-\beta\left(e^{\alpha x}-1\right)}, \quad x>0, \alpha>0, \beta>0,
$$

and The $c d f$ as follows:

$$
F(x)=1-e^{-\beta\left(e^{\alpha x}-1\right)} .
$$

This distribution should be denoted with two Gomp $\alpha$ and $\beta$ parameters. The research conducted by [1] indicated that a simple transformation relates the Gompertz distribution to a certain distribution in the family of distributions. A further research conducted by [7] showed that it is possible to get the maximum likelihood parameter estimates the Gompertz model. The study by [3] suggests the ways to apply it and provides a more recent survey that enables to better understand the model. At the same time, [19] made an attempt to reformulate the Gompertz mortality force and get an insight into the new formation relationship.

The analysis of the research by [18] enabled to trace the connections between the Weibull, the Gompertz, and other Type I extreme value distributions. Later, [9] managed to obtain a Bayesian prediction, mixing two-component lifetime model of Gompertz. In another study [10] derived a Bayesian record statistics analysis from the Gompertz model. A negative Gompertz distribution was presented by later, [11] who focused on the discussion of the negative aging parameter rate. A generalized three-parameter Gompertz distribution was presented by [8]), who provided a deep insight into the topic under investigation. Furthermore, [2]worked on the Gompertz model, and attempted to introduce a more generalized fourparameter version of the model that was referred to as a beta-Gompertz distribution. Also, the paper provides some commonly used distributions, including generalized and betaexponential Gompertz distributions as sub-models. [15] proposed a distribution of an exponentiated Weibull extension; however, it was modified. It was further generalized and discussed in the study by [8]. Author in [13] focused on the investigation and discussion of the obtained prediction intervals that are based on Gompertz doubly censored data. There are some cases make Progressive Hybrid Censored schemes (PHCS) difficult to apply when the failures may occur before time [21]. Some researchers estimated and predicted the Generalized Progressive Hybrid Censored Data for Gompertz Distribution [20]. Whoever Gompertz distribution was studied by many researchers such as [22].

The main objective of this paper, we assume that $X_{I}, X_{2}, \cdots, X_{n}$, is an ordered random sample of size $\mathrm{n}$ drawn from a population whose pdf, is $\operatorname{Gomp}(\alpha, \beta)$, which is defined by equation 1 , and that $Y_{1}, Y_{2}, \cdots, Y_{m}$. is a second independent random sample (of size $\mathrm{m}$ ) of future observations from the same distributions. Bayesian prediction bounds for the future observations $Y_{t}, Y_{z}, \cdots, Y_{m}$ in the presence of a single outlier of type $\beta \beta_{0}$ and $\beta+\beta_{0}$ are obtained. 
Observation is an outlier in the data set that is inconsistent with the data set remainder ([5]). Hence, a single $\beta \beta_{0}$, and $\beta+$ $\beta_{0}$ type outliers are present in the future Gompertz population sample. $\operatorname{Gomp}\left(\alpha, \beta \beta_{0}\right)$ is taken for a single type $\beta+\beta_{0}$ outlier of the $p d f$, while in the case of single type $\beta+\beta_{0}$ outlier the $p d f$ is taken $\operatorname{Gomp}\left(\alpha, \beta+\beta_{0}\right)$.

In the study, the bounds of the Bayesian prediction are received for the future $\operatorname{Gopm}(\alpha, \beta)$ distribution observations in the presence of a single outlier of type. It is considered that both parameters $\alpha$ and $\beta$ are unknown. The true value $(\beta, \alpha)$ uncertainty is measured through the function of the bivariate prior density that was discussed and applied with the same model in the research conducted by [10].Furthermore, the current research presupposes the construction of the predictive interval that will be used for the future observation with the presence of a single outlier of type with MCMC. The use of statistics will assist in illustrating and presenting the procedure.

In this article, Section II explains the Likelihood Function. After that Section III discuss the Posterior distribution. Moreover, Section IV clarify the Bayesian predication in the presence of outliers for future observations with two schemes $\beta \beta_{0}$ and $\beta+\beta_{0}$. Section $\mathrm{V}$ shows numerical example, which are consider the previous two schemes. In the final Section VI, we give the conclusion and opens future direction.

\section{LIKELIHOOD FUNCTION}

In this section, we assume $x_{1}, x_{2}, \cdots, x_{n}$ is an ordered random size $n$ sample from the $\operatorname{Gopm}(\alpha, \beta)$. The $p d f$ and $c d f$ are given be (1) and (2), respectively. Also, let $x_{1} \leq$ $x_{2} \leq \cdots \leq x_{k}$ be the $k$ smallest ordered observation, while $x_{r+1} \leq x_{r+2} \leq \cdots \leq x_{n}$, the $n-r$ largest ordered observations in the sample. The statistical analysis contains the application of only the remaining ordered observations, that is, $\underline{x}=\left(x_{k+1}, x_{s+2}, \cdots, x_{r}\right)$. Moreover, it is evident that when $\bar{k}=1$, the sample will be a Type-II right censored sample. A doubly censored sample pulled from population with pdf and cdf as given in (1) and (2) that likelihood function is given as follow:

$$
\begin{aligned}
L(\alpha, \beta ; \underline{x}) \propto & {\left[F_{X}\left(x_{k+1} ; \alpha, \beta\right)\right]^{k}\left[1-F_{X}\left(x_{r} ; \alpha, \beta\right)\right]^{n-r} } \\
& \times \prod_{i=k+1}^{r}\left[f_{X}\left(x_{i} ; \alpha, \beta\right)\right], x_{s+1} \geq 0 \\
= & (\alpha \beta)^{r-s}\left[1-\exp \left\{-\beta T_{1}\left(\alpha ; x_{k+1}\right)\right\}\right]^{k} \\
& \times \exp \left\{\alpha \sum_{i=s+1}^{r} x_{i}-\beta T_{2}(\underline{x} ; \alpha)\right\} .
\end{aligned}
$$

where

$$
\begin{aligned}
\left.T_{1}\left(\alpha ; x_{k+1}\right)\right) & =e^{\alpha x_{k+1}}-1 \\
T_{2}(\alpha ; \underline{x}) & =(n-r) e^{\alpha x_{r}}+\sum_{i=k+1}^{r} e^{\alpha x_{i}}-n+s .
\end{aligned}
$$

The Bayesian prediction tends to bound the future observations in the presence of a single outlier of type $\operatorname{Gomp}(\alpha, \beta)$ distribution when two parameters types $\alpha$ and $\beta$ are both dependent and unknown.

\section{THE POSTERIOR Distribution}

To obtain the joint posterior density of $\alpha$ and $\beta$, we use a bivariate prior density of the form:

$$
\pi(\alpha, \beta)=\pi_{1}(\alpha) \pi_{2}(\beta),
$$

where

$$
\pi_{1}(\alpha)=\frac{\gamma_{1}^{\eta_{1}}}{\Gamma\left(\eta_{1}\right)} \alpha^{\eta_{1}-1} e^{-\alpha \gamma_{1}},\left(\eta_{1}, \gamma_{1}>0\right)
$$

and

$$
\pi_{2}(\beta)=\frac{\gamma_{2}^{\eta_{2}}}{\Gamma\left(\eta_{2}\right)} \beta^{\eta_{2}-1} e^{-\beta \gamma_{2}}\left(\eta_{2}, \gamma_{2}>0\right)
$$

The paper assumes that the joint prior density for the parameter $\alpha$ and $\beta$ is the form (5) and presented by Jaheen [10] for the progressive censored data prediction from the Gompertz model and applied by [13] for the prediction Gompertz doubly censored data intervals.

The likelihood of the function presented by (3) and the function of the joint prior density presented by (5)as well as the function of the joint posterior density of $\alpha$ and $\beta$ is

$$
\pi^{*}(\alpha, \beta \mid \underline{x}) \quad=\frac{L(\alpha, \beta ; \underline{x}) \pi_{1}(\alpha) \pi_{2}(\beta)}{\int_{0}^{\infty} \int_{0}^{\infty} L(\alpha, \beta ; \underline{x}) \pi_{1}(\alpha) \pi_{2}(\beta) d \alpha d \beta} .(8)
$$

The joint posterior density function of $\alpha$ and $\beta$ given data can be written as

$$
\pi^{*}(\beta, \alpha, \mid \underline{x}) \propto h_{1}(\beta \mid \alpha, \text { data }) h_{2}(\alpha \mid \text { data }) h_{3}(\alpha, \beta \mid \text { data })
$$

where $h_{1}(\beta \mid \alpha$, data $)$ is a gamma density where the shape parameter $m=r-k+\eta_{1}$ and the scale parameter is $\gamma_{1}+T_{2}(\alpha ; \underline{x})$. At the same time, $h_{2}(\alpha \mid$ data $)$ is a proper density function of the form

$$
\begin{aligned}
h_{2}(\alpha \mid \text { data }) \propto \quad & \frac{1}{\left[\gamma_{1}+T_{2}(\alpha ; \underline{x})\right]^{m}} \alpha^{r-k+\eta_{2}-1} \\
& e^{-\alpha\left(\frac{1}{\gamma_{2}}-\sum_{i=k+1}^{r} x_{i}\right)}
\end{aligned}
$$

and $h_{3}(\alpha, \beta \mid$ data $\left.)\right)$ is given by

$$
h_{3}(\alpha, \beta \mid \text { data })=\left[1-e^{-\beta T_{1}\left(\alpha ; x_{k+1}\right)}\right]^{s} .
$$

From equation (8) and it enables to see that a simple closed form cannot express the equation. Therefore, the Bayes estimators of the parameter $\alpha$ and $\beta$ cannot be received in simple closed forms. Hence, the paper suggests the approximation (9) by applying the importance sampling technique that is also presented by [14]. The importance sampling details are presented below.

In this paper, we used the importance sampling procedure to calculate the Bayes estimates for $\alpha, \beta$ as well as any function of the parameters $g(\alpha, \beta)$. Moreover, the Algorithm 1 (presented below) is used to generate $\alpha$ and $\beta$ from the posterior density function (7).

\section{Algorithm 1:}


Step 1 : Start with an $\left(\alpha^{0} ; \beta^{0}\right)$.

Step $2:$ set $\mathrm{t}=1$.

Step 3 : Generate $\alpha^{t}$ from $h_{2}(\alpha \mid$ data $)$ using the method developed by [12] with the $N\left(\alpha^{t-1}, \sigma\right)$ proposal distribution, where $\sigma^{2}$ is the variance of the parameter $\alpha$.

Step 4 : Generate $\beta^{t}$ from gamma distribution with pdf $h_{2}(\beta \mid \alpha$, data $)$.

Step 5 : Put $\mathrm{t}=\mathrm{t}+1$.

Step 6 : Repeat steps 3-5 $M$ times to obtain $\left\{\left(\alpha^{t}, \beta^{t}\right), t=\right.$ $1,2, \cdots, M\}$.

The approximate Bayes are applied to estimate any function of the parameters say $g(\alpha, \beta)$ under the squared functions of error loss using the procedure of importance sampling, as shown below:

$$
\hat{g}_{B S}(\alpha, \beta)=\frac{\sum_{i=1}^{M} g\left(\alpha_{i}, \beta_{i}\right) g_{3}\left(\alpha_{i}, \beta_{i} \mid \text { data }\right)}{\sum_{i=M_{0}}^{M} g_{3}\left(\alpha_{i}, \beta_{i} \mid \text { data }\right)},
$$

\section{Bayesian Prediction in the Presence of a Single OUTLIER FOR FUTURE OBSERVATIONS}

The section introduces the prediction of the future observations in the presence of a single outlier. Also, it is assumed that $X_{1}, X_{2}, \cdots, X_{n}$ is a random size $n$ sample drawn from the $\operatorname{Gomp}(\alpha, \beta)$ population, where the $p d f$ is presented by (1). Let us assume that $Y_{1}, Y_{2}, \cdots, Y_{m}$ is a second, independent, unobserved size $m$ sample received from the same population. This sample is the future sample, and the aim of the study is to get Bayesian prediction bounds for the $s^{\text {th }}$ oncoming observation $Y_{s}, s=1,2, \cdots, m$ in the presence of a single outlier.

In the case of the size $m$ sample, let $Y_{s}$ be the $s^{\text {th }}$ ordered lifetime, $1 \leq s \leq m$. Then the $Y_{s}$ density function for a given $\theta$ in the presence of a single outlier is of the form $f=f(y \mid \theta)$ and $F=F(y \mid \theta)$ are the distribution and density functions of all $y_{s}$ which are not referred to be outliers as $f^{*}=f^{*}(y \mid \theta)$ and $F^{*}=F^{*}(y \mid \theta)$ are those of an outlier ([4]). The $f^{*}$ and $F^{*}$ functions are received for the $\operatorname{Gomp}(\alpha, \beta)$ model through the replacement of parameter $\beta$ by $\beta \beta_{0}$ or $\beta+\beta_{0}$ depending on the outlier type.

$$
\begin{aligned}
f\left(y_{s} \mid \theta\right)= & D(s)\left[(s-1) F^{s-2}(1-F)^{m-s} F^{\star} f\right. \\
& +(m-s) F^{s-1}(1-F)^{m-s-1}\left(1-F^{\star}\right) f \\
& \left.+F^{s-1}(1-F)^{m-s} f^{\star}\right],
\end{aligned}
$$

where

$$
D(s)=\left(\begin{array}{c}
m-1 \\
s-1
\end{array}\right)
$$

\section{A. Outliers of type $\beta \beta_{0}$}

The $Y_{s}$ density function, in the presence of a single outlier of type $\beta \beta_{0}$, in the $\operatorname{Gomp}(\alpha, \beta)$ case may be received through the substituting of (1) and (2) for $f$ and $F$ in (13). The $f^{*}$ and $F^{*}$ values presented by (1) and (2), after the replacement of $\beta$ by $\beta \beta_{0}$. It is possible to simplify the density function implementing the $p d f g_{1}\left(y_{2} \mid \alpha, \beta\right)$, where the $c d f G_{1}\left(y_{s} \mid \alpha, \beta\right)$ is given as follows:

$$
\begin{aligned}
g_{1}\left(y_{s} \mid \alpha, \beta\right)=D(s) \alpha \beta e^{\alpha y_{s}}\left[\left(m+\beta_{0}-s\right) \sum_{j=0}^{s-1} A_{1 j}\left(y_{s}\right)\right. \\
\left.+(s-1) \sum_{j=0}^{s-2} A_{2 j}\left(y_{s}\right)\right], \quad y_{s}>0,
\end{aligned}
$$

where

$$
\begin{aligned}
A_{1 j}\left(y_{s}\right)= & a_{1 j}(s) \exp \left\{-\beta \omega_{j}(s) \phi\left(y_{s} ; \alpha\right)\right\}, \\
A_{2 j}\left(y_{s}\right)= & a_{2 j}(s)\left[\exp \left\{-\beta \omega_{1 j}(s) \phi\left(y_{s} ; \alpha\right)\right\}\right. \\
& \left.-\exp \left\{-\beta \omega_{j+1}(s) \phi\left(y_{s} ; \alpha\right)\right\}\right], \\
\phi\left(y_{s} ; \alpha\right)= & \left(e^{\alpha y_{s}}-1\right) \\
\omega_{j}(s)= & m-s+\beta_{0}+j, \\
\omega_{1 j}(s)= & m-s+j+1
\end{aligned}
$$

and for $\ell=1,2$,

$$
a_{\ell j}(s)=(-1)^{j}\left(\begin{array}{c}
s-\ell \\
j
\end{array}\right) .
$$

and the pdf $g_{1}\left(y_{s} \mid \alpha, \beta\right)$ the $\operatorname{cdf} G_{1}\left(y_{s} \mid \alpha, \beta\right)$ is given by

$$
\begin{aligned}
G_{1}\left(y_{s} \mid \alpha, \beta\right)=D(s)\left[\left(m+\beta_{0}-s\right) \sum_{j=0}^{s-1} A_{1 j}^{*}\left(y_{s}\right)\right. \\
\left.+(s-1) \sum_{j=0}^{s-2} A_{2 j}^{*}\left(y_{s}\right)\right], \quad y_{s}>0
\end{aligned}
$$

where

$$
\begin{aligned}
A_{1 j}^{*}\left(y_{s}\right) & =\frac{a_{1 j}(s)}{\omega_{j}(s)} F\left(y_{s} ; \alpha, \beta \omega_{j}(s)\right), \\
A_{2 j}^{*}\left(y_{s}\right) & =\frac{a_{2 j}(s)}{\omega_{1 j}(s)} F\left(y_{s} ; \alpha, \beta \omega_{1 j}(s)\right) \\
& -\frac{a_{2 j}(s)}{\omega_{j+1}(s)} F\left(y_{s} ; \alpha, \beta \omega_{j+1}(s)\right) .
\end{aligned}
$$

The Bayesian predictive density of $y_{s}, s=1,2, \cdots, m$ given $\underline{x}$ is represented by

$$
g_{1}^{*}\left(y_{s} \mid \underline{x}\right)=\int_{0}^{\infty} \int_{0}^{\infty} g_{1}\left(y_{s} \mid \alpha, \beta\right) \pi^{*}(\alpha, \beta \mid \underline{x}) d \alpha d \beta .
$$

The Bayesian predictive distribution function of $y_{s}, s=$ $1,2, \cdots, m$ given $\underline{x}, \alpha$ and $\beta$ is given by

$$
G_{1}^{*}\left(y_{s} \mid \underline{x}\right)=\int_{0}^{\infty} \int_{0}^{\infty} G_{1}\left(y_{s} \mid \alpha, \beta\right) \pi^{*}(\alpha, \beta \mid \underline{x}) d \alpha d \beta .
$$

Supposing that $\left\{\left(\alpha_{i}, \beta_{i}\right) ; i=1,2, \cdots, M\right\}$ are MCMC samples received from $\pi^{*}(\alpha, \beta \mid \underline{x})$, it is possible to get the 
simulation consistent estimators of $g_{1}^{*}\left(y_{s} \mid \underline{x}\right)$ and $G^{*}\left(y_{s} \mid \underline{x}\right)$ can be obtained as

$$
\hat{g}_{1}^{*}\left(y_{s} \mid \underline{x}\right)=\sum_{i=1}^{M} g_{1}\left(y_{s} \mid \alpha_{i}, \beta_{i}\right) h_{i}
$$

and

$$
\hat{G}_{1}^{*}\left(y_{s} \mid \underline{x}\right)=\sum_{i=1}^{M} G_{1}\left(y_{s} \mid \alpha_{i}, \beta_{i}\right) h_{i}
$$

where

$$
h_{i}=\frac{h_{3}\left(\alpha_{i}, \beta_{i}\right)}{\sum_{i=1}^{M} h_{3}\left(\alpha_{i}, \beta_{i}\right)} ; \quad i=1,2, \cdots, M .
$$

A $(1-\tau) 100 \%$ Bayesian prediction interval for $Y_{s}$ is as follows: $P\left[L(\underline{x}) \leq Y_{s} \leq U(\underline{x})\right]=1-\tau$, where $L(\underline{x})$ and $U(\underline{x})$ are the lower and the upper bounds for $y_{s}$, $s=1,2, \cdots, m$. Thus, equating of (23) $1-\frac{\tau}{2}$ and $\frac{\tau}{2}$, enables to get the following:

$$
P\left[Y_{s} \geq L(\underline{x}) \mid \underline{x}\right]=1-\frac{\tau}{2} \Rightarrow \hat{G}_{1}^{*}(L(\underline{x}) \mid \underline{x})=\frac{\tau}{2}
$$

and

$$
P\left[Y_{s} \leq U(\underline{x}) \mid \underline{x}\right]=\frac{\tau}{2} \Rightarrow \hat{G}_{1}^{*}(U(\underline{x}) \mid \underline{x})=1-\frac{\tau}{2} .
$$

\section{B. Type $\beta+\beta_{0}$ Outliers}

The $y_{s}$ density function, in the presence of a single outlier of type $\beta+\beta_{0}$, in the $\operatorname{Gomp}(\alpha, \beta)$ case, can be received through the substituting of (1) and (2) for $F$ and $f$ in (3). The $F^{*}$ and $f^{*}$ are presented by (1) and (2) after the replacement of $\beta$ by $\beta+\beta_{0}$. Consequently, the density begins to form:

$$
\begin{array}{r}
g_{2}\left(y_{s} \mid \alpha, \beta\right)=D(s) e^{\alpha y_{s}}\left[\left(\beta(m-s+1)+\beta_{0}\right) \sum_{j=0}^{s-1} B_{1 j}\left(y_{s}\right)\right. \\
\left.+\beta(s-1) \sum_{j=0}^{s-2} B_{2 j}\left(y_{s}\right)\right], y_{s}>0, \quad(27)
\end{array}
$$

where

$$
\begin{aligned}
B_{1 j}\left(y_{s}\right) & =a_{1 j}(s) \exp \left\{-\left[\beta \omega_{1 j}(s)+\beta_{0}\right] \phi\left(y_{s} ; \alpha\right)\right\} \\
B_{2 j}\left(y_{s}\right) & =a_{2 j}(s)\left[\exp \left\{-\beta \omega_{1 j}(s) \phi\left(y_{s} ; \alpha\right)\right\}\right. \\
& \left.-\exp \left\{-\left[\beta \omega_{1(j+1)}(s)+\beta_{0}\right] \phi\left(y_{s} ; \alpha\right)\right\}\right],
\end{aligned}
$$

$\phi\left(y_{s} ; \alpha\right) \omega_{1 j}(s)$ are given in (16) and $a_{\ell j}(s), a_{2} j(s)$ is given for $\ell=1,2$, respectively, by (17).

The cdf corresponding to the pdf $g_{2}\left(y_{s} \mid \alpha, \beta\right)$ is presented by

$$
\begin{aligned}
G_{2}\left(y_{s} \mid \alpha, \beta\right)=D(s) & {\left[\left(\beta(m-s+1)+\beta_{0}\right) \sum_{j=0}^{s-1} B_{1 j}^{*}\left(y_{s}\right)\right.} \\
& \left.+\beta(s-1) \sum_{j=0}^{s-2} B_{2 j}^{*}\left(y_{s}\right)\right], y_{s}>0
\end{aligned}
$$

where

$$
\begin{aligned}
B_{1 j}^{*}\left(y_{s}\right) & =\frac{a_{1 j}(s)}{\beta \omega_{1 j}(s)+\beta_{0}} F\left(y_{s} ; \alpha, \beta \omega_{1 j}(s)+\beta_{0}\right), \\
B_{2 j}^{*}\left(y_{s}\right) & =\frac{a_{2 j}(s)}{\beta \omega_{1 j}(s)} F\left(y_{s} ; \alpha, \beta \omega_{1 j}(s)\right) \\
& -\frac{a_{2 j}(s)}{\beta \omega_{1(j+1)}(s)+\beta_{0}} F\left(y_{s} ; \alpha, \beta \omega_{1(j+1)}(s)+\beta_{0}\right),
\end{aligned}
$$

where $F\left(y_{s} ; \alpha, \beta m+\beta_{0}\right)$ is given by(2).

The Bayesian predictive distribution function of $y_{s}, s=$ $1,2, \cdots, m$ given $\underline{x}, \alpha$ and $\beta$ is given by

$$
g_{2}^{*}\left(y_{s} \mid \underline{x}\right)=\int_{0}^{\infty} \int_{0}^{\infty} g_{2}\left(y_{s} \mid \alpha, \beta\right) \pi^{*}(\alpha, \beta \mid \underline{x}) d \alpha d \beta
$$

and the predictive cdf of $y_{s}, G_{2}^{*}\left(y_{s} \mid \underline{x}\right)$ is given by

$$
G_{2}^{*}\left(y_{s} \mid \underline{x}\right)=\int_{0}^{\infty} \int_{0}^{\infty} G_{2}\left(y_{s} \mid \alpha, \beta\right) \pi^{*}(\alpha, \beta \mid \underline{x}) d \alpha d \beta,
$$

where $G_{2}\left(y_{s} \mid \alpha, \beta\right)$ is given by (29) and $\pi^{*}(\alpha, \beta \mid \underline{x})$ is given by (9). It is evident that it is impossible to express (31) and (32) in closed form. Therefore, they cannot be analytically evaluated.

The use of MCMC samples $\left\{\left(\alpha_{i}, \beta_{i}\right), i=1,2, \cdots, M\right\}$, enable the obtaining of $g_{2}^{*}\left(y_{s} \mid \underline{x}\right)$ and $G_{2}^{*}\left(y_{s} \mid \underline{x}\right)$ simulation consistent estimator, as follows:

$$
\hat{g}_{2}^{*}\left(y_{s} \mid \underline{x}\right)=\sum_{i=1}^{M} g_{2}\left(y_{s} \mid \alpha_{i}, \beta_{i}\right) h_{i},
$$

and

$$
\hat{G}_{2}^{*}\left(y_{s} \mid \underline{x}\right)=\sum_{i=1}^{M} G_{2}\left(y_{s} \mid \alpha_{i}, \beta_{i}\right) h_{i},
$$

Where $h_{i}$ is given by (24). It is essential to highlight that it is possible to use the same MCMC samples $\left\{\left(\alpha_{i}, \beta_{i}\right), i=\right.$ $1,2, \cdots, M\}$, to compute $\hat{g}_{2}^{*}\left(y_{s} \mid \underline{x}\right)$ and $\hat{G}_{2}^{*}\left(y_{s} \mid \underline{x}\right)$ for all $y_{s}$. Also, A $(1-\tau) 100 \%$ Bayesian prediction intervals for is $P\left[L(\underline{x}) \leq Y_{s} \leq U(\underline{x})\right]=1-\tau$ where $L(\underline{x})$ and $U(\underline{x})$ are lower and upper $y_{s}$ Bayesian prediction bounds. Hence, it is possible to get the lower and upper Bayesian prediction bounds, $L(\underline{x})$ and $U(\underline{x})$, for $y_{s}, s=1,2, \cdot, m$ through solving the following two nonlinear equations.

$$
P\left[Y_{s} \geq L(\underline{x}) \mid \underline{x}\right]=1-\frac{\tau}{2} \Rightarrow \hat{G}_{2}^{*}(L(\underline{x}) \mid \underline{x})=\frac{\tau}{2}
$$

and

$$
P\left[Y_{s} \leq U(\underline{x}) \mid \underline{x}\right]=\frac{\tau}{2} \Rightarrow \hat{G}_{2}^{*}(U(\underline{x}) \mid \underline{x})=1-\frac{\tau}{2} .
$$

It is possible to solve the two nonlinear equations (35) and (36) through the use of an iterative method to receive the lower and upper Bayesian prediction bounds for $y_{s} ; s=1,2, \cdots, m$. 


\section{NUMERICAL EXAMPLE}

Example 1. This example shows a doubly Type-II censored sample, $x_{(s+1)}, x_{(s+2)}, \cdots, x_{(r)}$, that is received through the application of the following steps:

1 - For the hyperparameters given values $\eta_{1}=1.2$ and $\gamma_{1}=1.8$ a generated value of $\alpha=0.860986$ is received from the prior distribution with pdf (6).

2 - For the hyperparameters given values $\eta_{2}=1.4$ and $\gamma_{2}=1.7$ a generated value of $\beta=0.409442$ is received from the prior distribution with pdf (7).

3 - The use of the generated values of $\alpha$ and $\beta$ from two prior steps, enables to generate a sample of size $n=$ 30 from the $\operatorname{Gomp}(\alpha, \beta)$ distribution with $p d f$, that is represented by (2).

4 - The application of some sorting routine, assists in obtaining a doubly Type-II censored different value sample of size $r=20,25,30$ and $k=0,5,10$ from the $\operatorname{Gomp}(\alpha, \beta)$ distribution, where the deferment value of $r$ and $k$ is presented in Tables I, II and III.

5 - $\quad$ Generate $\left(\alpha_{i}, \beta_{i}\right), i=1,2, \cdots, M$, through the use of MCMC shown in Algorithm 1.

6 - The above generated doubly Type-II censored size $(r-s)$ sample, the $95 \%$ Bayesian prediction links to the future ordered values, $y_{(1)}, y_{(2)}, \cdots, y_{(m)}, m=$ 5 in the single types $\beta \beta 0$ outliers, enable a numerical calculation through solving the equations (25) and (26).

Let us assume that we have one more size $m=5$ sample in the presence of a single outlier of type $\beta \beta_{0}$. Hence, for the given $\beta_{0}$ values we seek to receive $95 \%$ Bayesian prediction bounds for $y_{1}$ to $y_{5}$ of the failure future sample times. Tables I, II and III represents these bounds with the corresponding $\beta_{0}$ values.

Example 2. The $95 \%$ Bayesian prediction interval for a future unobserved $y_{1}$ to $y_{5}$, which are the failure times in the future size 5 sample in the presence of a single outlier of type $\beta+\beta_{0}$ can be obtained on the basis of a generated doubly Type-II censored sample of sizen from the Gomp $(\alpha, \beta)$ distribution. Same different $\eta_{1}, \gamma_{1}, \eta_{2}, \gamma_{1}$ hyper-parameter values and the same data set is presented in Example 1. Hence, these bounds with the corresponding $n=30, r=20,25,20$ and $k=n-r$ and $\beta_{0}$ values are shown in Tables IV, V and VI.

\section{CONCLUSION}

The study investigated and discussed the single $\beta \beta_{0}$ and $\beta+\beta_{0}$ type outliers through the application of the predictive distribution function. Hence, the Bayesian prediction intervals in the case of future homogeneous case observations can be received by $\beta_{0}=1$ in (18) or $\beta_{0}=0$ in (29).

However, it is impossible in the no outlier case. The Gibbs sampling technique was applied to generate MCMC samples. Afterwards, the importance sampling methodology was used to compute the Bayesian prediction problems in the presence of a single outlier of both type. It is essential to highlight that the Bayesian prediction intervals are shorter for $y_{1}$ and larger for the Bayesian prediction intervals for $y_{5}$ due to the increase of $\beta_{0}$ value.
TABLE I. $\quad 95 \%$ BAYESIAN PREDICTION INTERVALS FOR $y_{1}, \cdots, y_{5}$ IN THE PRESENCE OF A SINGLE OUTLIER OF TYPE $\beta \beta_{0}$, WHERE $n=30, r=20, k=10$. NOTE: OBS. IS OBSERVATIONS PP IS POINT PREDICTORS, LB IS LOWER BOUND, UB IS UPPER BOUND, CP IS Coverage Percentages.

\begin{tabular}{|l|l|l|l|l|l|l|}
\hline$\beta_{0}$ & Obs & $y_{1}$ & $y_{2}$ & $y_{3}$ & $y_{4}$ & $y_{5}$ \\
\hline 1 & PP & 0.492721 & 0.933387 & 1.35569 & 1.80134 & 2.37285 \\
\cline { 2 - 7 } & LB & 0.017663 & 0.177097 & 0.460092 & 0.824819 & 1.29551 \\
& UB & 1.39901 & 1.88431 & 2.30933 & 2.76994 & 3.43655 \\
\cline { 2 - 7 } & Length & 1.38135 & 1.70721 & 1.84924 & 1.94513 & 2.14104 \\
\cline { 2 - 7 } & CP & $95.77 \%$ & $95.62 \%$ & $95.03 \%$ & $94.79 \%$ & $93.86 \%$ \\
\hline 2 & PP & 0.428709 & 0.836901 & 1.24704 & 1.69552 & 2.285 \\
\cline { 2 - 7 } & LB & 0.014736 & 0.151347 & 0.403924 & 0.743443 & 1.19871 \\
& UB & 1.2507 & 1.73927 & 2.1873 & 2.68116 & 3.38701 \\
\cline { 2 - 7 } & Length & 1.23597 & 1.58792 & 1.78338 & 1.93772 & 2.1883 \\
\cline { 2 - 7 } & CP & $95.17 \%$ & $95.84 \%$ & $95.91 \%$ & $96.12 \%$ & $95.65 \%$ \\
\hline 3 & PP & 0.379907 & 0.77606 & 1.19357 & 1.65873 & 2.26774 \\
\cline { 2 - 7 } & LB & 0.012642 & 0.13457 & 0.370458 & 0.700365 & 1.15708 \\
& UB & 1.13273 & 1.66262 & 2.15497 & 2.67353 & 3.38666 \\
\cline { 2 - 7 } & Length & 1.12009 & 1.52805 & 1.78451 & 1.97316 & 2.22958 \\
\cline { 2 - 7 } & CP & $94.19 \%$ & $95.73 \%$ & $96.4 \%$ & $96.77 \%$ & $96.31 \%$ \\
\hline 4 & PP & 0.34137 & 0.735004 & 1.16389 & 1.64286 & 2.26251 \\
\cline { 2 - 7 } & LB & 0.011069 & 0.122535 & 0.34753 & 0.6731 & 1.13514 \\
& UB & 1.03625 & 1.62423 & 2.14778 & 2.67299 & 3.38665 \\
\cline { 2 - 7 } & Length & 1.02518 & 1.5017 & 1.80025 & 1.99989 & 2.25152 \\
\cline { 2 - 7 } & CP & $92.88 \%$ & $95.61 \%$ & $96.64 \%$ & $97.25 \%$ & $96.6 \%$ \\
\hline 5 & PP & 0.310111 & 0.705889 & 1.14598 & 1.63502 & 2.2605 \\
\cline { 2 - 7 } & LB & 0.009844 & 0.113362 & 0.33055 & 0.654265 & 1.12263 \\
& UB & 0.955644 & 1.60625 & 2.14633 & 2.67296 & 3.38665 \\
\cline { 2 - 7 } & Length & 0.945801 & 1.49289 & 1.81578 & 2.01869 & 2.26402 \\
\cline { 2 - 7 } & CP & $91.31 \%$ & $95.49 \%$ & $96.86 \%$ & $97.55 \%$ & $96.77 \%$ \\
\hline
\end{tabular}

TABLE II. $95 \%$ BAYESIAN PREDICTION INTERVALS FOR $y_{1}, \cdots, y_{5}$ IN THE PRESENCE OF A SINGLE OUTLIER OF TYPE $\beta \beta_{0}$, WHERE $n=30, r=25, k=5$. NOTE:OBS. IS OBSERVATIONS PP IS POINT PREDICTORS, LB IS LOWER BOUND, UB IS UPPER BOUND, CP IS Coverage Percentages.

\begin{tabular}{|l|l|l|l|l|l|l|}
\hline$\beta_{0}$ & Obs & $y_{1}$ & $y_{2}$ & $y_{3}$ & $y_{4}$ & $y_{5}$ \\
\hline 1 & PP & 0.480366 & 0.912016 & 1.3272 & 1.76659 & 2.33151 \\
\cline { 2 - 7 } & LB & 0.017118 & 0.171888 & 0.447624 & 0.804559 & 1.26715 \\
& UB & 1.3691 & 1.84794 & 2.26819 & 2.72426 & 3.38507 \\
\cline { 2 - 7 } & Length & 1.35198 & 1.67605 & 1.82057 & 1.9197 & 2.11793 \\
\cline { 2 - 7 } & CP & $95.69 \%$ & $95.69 \%$ & $95.12 \%$ & $95.04 \%$ & $94.49 \%$ \\
\hline 2 & PP & 0.417723 & 0.817269 & 1.22021 & 1.66216 & 2.24463 \\
\cline { 2 - 7 } & LB & 0.014281 & 0.146862 & 0.392804 & 0.724791 & 1.17186 \\
& UB & 1.22303 & 1.7047 & 2.14747 & 2.63631 & 3.33594 \\
\cline { 2 - 7 } & Length & 1.20875 & 1.55784 & 1.75466 & 1.91152 & 2.16407 \\
\cline { 2 - 7 } & CP & $94.88 \%$ & $95.76 \%$ & $95.86 \%$ & $96.25 \%$ & $96.03 \%$ \\
\hline 3 & PP & 0.370002 & 0.757583 & 1.16762 & 1.6259 & 2.22759 \\
\cline { 2 - 7 } & LB & 0.012251 & 0.130561 & 0.360161 & 0.682593 & 1.13091 \\
& UB & 1.10696 & 1.62904 & 2.11549 & 2.62875 & 3.33559 \\
\cline { 2 - 7 } & Length & 1.09471 & 1.49848 & 1.75533 & 1.94616 & 2.20467 \\
\cline { 2 - 7 } & CP & $94 . \%$ & $95.41 \%$ & $96.21 \%$ & $96.84 \%$ & $96.5 \%$ \\
\hline 4 & PP & 0.332341 & 0.717339 & 1.13846 & 1.61028 & 2.22243 \\
\cline { 2 - 7 } & LB & 0.010726 & 0.118871 & 0.337806 & 0.655895 & 1.10933 \\
& UB & 1.01211 & 1.59117 & 2.10838 & 2.62822 & 3.33558 \\
\cline { 2 - 7 } & Length & 1.00139 & 1.4723 & 1.77057 & 1.97233 & 2.22625 \\
\cline { 2 - 7 } & CP & $92.5 \%$ & $95.15 \%$ & $96.33 \%$ & $97.33 \%$ & $96.79 \%$ \\
\hline 5 & PP & 0.30181 & 0.688818 & 1.12087 & 1.60256 & 2.22046 \\
\cline { 2 - 7 } & LB & 0.009539 & 0.109964 & 0.321256 & 0.637456 & 1.09703 \\
& UB & 0.93294 & 1.57343 & 2.10694 & 2.62818 & 3.33558 \\
\cline { 2 - 7 } & Length & 0.923401 & 1.46347 & 1.78568 & 1.99073 & 2.23855 \\
\cline { 2 - 7 } & CP & $90.53 \%$ & $95.11 \%$ & $96.51 \%$ & $97.58 \%$ & $96.94 \%$ \\
\hline
\end{tabular}

\section{ACKNOWLEDGMENT}

The authors extend their appreciation to the Deanship of Scientific research at Majmaah University for funding this work through research project No. R-1441-85.

\section{REFERENCES}

[1] JC. Ahuja and SW Nash, The Generalized Gompertz-Verhulst Family of Distributions, Sankhya, 1967.

[2] J. Ali, T. Saeid, and A. Morad, The beta-Gompertz distribution, Revista Colombiana de Estadistica, 2014. 
TABLE III. $\quad 95 \%$ BAYESIAN PREDICTION INTERVALS FOR $y_{1}, \cdots, y_{5}$ IN THE PRESENCE OF A SINGLE OUTLIER OF TYPE $\beta \beta_{0}$, WHERE $n=30, r=30, k=0$. NOTE:OBS. IS OBSERVATIONS PP IS POINT PREDICTORS, LB IS LOWER BOUND, UB IS UPPER BOUND, CP IS COVERAge Percentages.

\begin{tabular}{|l|l|l|l|l|l|l|}
\hline$\beta_{0}$ & Obs & $y_{1}$ & $y_{2}$ & $y_{3}$ & $y_{4}$ & $y_{5}$ \\
\hline 1 & PP & 0.475422 & 0.902867 & 1.31418 & 1.74964 & 2.30966 \\
\cline { 2 - 7 } & LB & 0.016929 & 0.170029 & 0.442908 & 0.796326 & 1.25459 \\
& UB & 1.35561 & 1.8302 & 2.24682 & 2.69903 & 3.35434 \\
\cline { 2 - 7 } & Length & 1.33868 & 1.66017 & 1.80392 & 1.9027 & 2.09975 \\
\cline { 2 - 7 } & CP & $95.56 \%$ & $95.69 \%$ & $95.21 \%$ & $95.11 \%$ & $94.63 \%$ \\
\hline 2 & PP & 0.413396 & 0.809015 & 1.20817 & 1.64613 & 2.22353 \\
\cline { 2 - 7 } & LB & 0.014124 & 0.145269 & 0.388644 & 0.717329 & 1.16018 \\
& UB & 1.21088 & 1.68822 & 2.12713 & 2.61182 & 3.30561 \\
\cline { 2 - 7 } & Length & 1.19675 & 1.54295 & 1.73849 & 1.89449 & 2.14543 \\
\cline { 2 - 7 } & CP & $94.81 \%$ & $95.63 \%$ & $95.72 \%$ & $96.19 \%$ & $95.98 \%$ \\
\hline 3 & PP & 0.366148 & 0.7499 & 1.15607 & 1.61019 & 2.20664 \\
\cline { 2 - 7 } & LB & 0.012116 & 0.129143 & 0.356335 & 0.675541 & 1.11961 \\
& UB & 1.09588 & 1.61323 & 2.09543 & 2.60433 & 3.30526 \\
\cline { 2 - 7 } & Length & 1.08376 & 1.48409 & 1.73909 & 1.92878 & 2.18565 \\
\cline { 2 - 7 } & CP & $93.81 \%$ & $95.25 \%$ & $95.95 \%$ & $96.84 \%$ & $96.49 \%$ \\
\hline 4 & PP & 0.328865 & 0.710044 & 1.12718 & 1.59472 & 2.20152 \\
\cline { 2 - 7 } & LB & 0.010608 & 0.117579 & 0.334211 & 0.649105 & 1.09822 \\
& UB & 1.00191 & 1.57569 & 2.08838 & 2.6038 & 3.30526 \\
\cline { 2 - 7 } & Length & 0.991305 & 1.45811 & 1.75417 & 1.95469 & 2.20703 \\
\cline { 2 - 7 } & CP & $92.35 \%$ & $94.94 \%$ & $96.15 \%$ & $97.25 \%$ & $96.81 \%$ \\
\hline 54 & PP & 0.298642 & 0.681802 & 1.10976 & 1.58707 & 2.19956 \\
\cline { 2 - 7 } & LB & 0.009434 & 0.108767 & 0.317832 & 0.630847 & 1.08604 \\
& UB & 0.923483 & 1.55811 & 2.08696 & 2.60376 & 3.30526 \\
\cline { 2 - 7 } & Length & 0.914049 & 1.44934 & 1.76912 & 1.97292 & 2.21922 \\
\cline { 2 - 7 } & CP & $90.22 \%$ & $94.88 \%$ & $96.31 \%$ & $97.43 \%$ & $96.93 \%$ \\
\hline
\end{tabular}

TABLE IV. $\quad 95 \%$ BAYESIAN PREDICTION INTERVALS FOR $y_{1}, \cdots, y_{5}$ IN THE PRESENCE OF A SINGLE OUTLIER OF TYPE $\beta+\beta_{0}$, WHERE $n=30, r=20, k=10$. NOTE: OBS. IS OBSERVATIONS PP IS POINT PREDICTORS, LB IS LOWER BOUND, UB IS UPPER BOUND, CP IS Coverage Percentages.

\begin{tabular}{|l|l|l|l|l|l|l|}
\hline$\beta_{0}$ & Obs & $y_{1}$ & $y_{2}$ & $y_{3}$ & $y_{4}$ & $y_{5}$ \\
\hline 0 & PP & 0.314436 & 0.624792 & 0.947281 & 1.3117 & 1.80927 \\
\cline { 2 - 7 } & LB & 0.010012 & 0.103158 & 0.280504 & 0.530439 & 0.8847 \\
& UB & 0.966839 & 1.36921 & 1.7411 & 2.15974 & 2.78612 \\
\cline { 2 - 7 } & Length & 0.956827 & 1.26605 & 1.46059 & 1.6293 & 1.90142 \\
\cline { 2 - 7 } & CP & $95.48 \%$ & $95.68 \%$ & $95.32 \%$ & $95.39 \%$ & $95.04 \%$ \\
\hline 1 & PP & 0.249322 & 0.524206 & 0.833376 & 1.20209 & 1.72088 \\
\cline { 2 - 7 } & LB & 0.00759 & 0.081351 & 0.230135 & 0.452727 & 0.787286 \\
& UB & 0.792205 & 1.20258 & 1.61103 & 2.07199 & 2.7386 \\
\cline { 2 - 7 } & Length & 0.784615 & 1.12123 & 1.3809 & 1.61926 & 1.95131 \\
\cline { 2 - 7 } & CP & $94.01 \%$ & $94.96 \%$ & $95.6 \%$ & $96.61 \%$ & $96.6 \%$ \\
\hline \multirow{1}{*}{2} & PP & 0.206843 & 0.474838 & 0.794469 & 1.17926 & 1.71243 \\
\cline { 2 - 7 } & LB & 0.006111 & 0.069507 & 0.205725 & 0.420648 & 0.757922 \\
& UB & 0.67229 & 1.14618 & 1.59745 & 2.07048 & 2.73858 \\
\cline { 2 - 7 } & Length & 0.666179 & 1.07668 & 1.39172 & 1.64983 & 1.98066 \\
\cline { 2 - 7 } & CP & $91.36 \%$ & $94.35 \%$ & $95.9 \%$ & $97.18 \%$ & $97.02 \%$ \\
\hline 3 & PP & 0.176862 & 0.446821 & 0.777449 & 1.17201 & 1.71067 \\
\cline { 2 - 7 } & LB & 0.005115 & 0.06181 & 0.190618 & 0.402904 & 0.746022 \\
& UB & 0.584482 & 1.12971 & 1.59639 & 2.07045 & 2.73858 \\
\cline { 2 - 7 } & Length & 0.579367 & 1.0679 & 1.40577 & 1.66755 & 1.99256 \\
\cline { 2 - 7 } & CP & $87.7 \%$ & $94.34 \%$ & $96.26 \%$ & $97.31 \%$ & $97.2 \%$ \\
\hline 4 & PP & 0.154538 & 0.429346 & 0.768791 & 1.16911 & 1.71014 \\
\cline { 2 - 7 } & LB & 0.004398 & 0.056299 & 0.180133 & 0.391883 & 0.740829 \\
& UB & 0.517254 & 1.12552 & 1.59631 & 2.07045 & 2.73858 \\
\cline { 2 - 7 } & Length & 0.512856 & 1.06922 & 1.41618 & 1.67857 & 1.99775 \\
\cline { 2 - 7 } & CP & $84.05 \%$ & $94.45 \%$ & $96.45 \%$ & $97.45 \%$ & $97.28 \%$ \\
\hline
\end{tabular}

[3] E.K. AL-Hussaini, G.R. AL-Dayian, and S.A. Adham, On Finite Mixture of Two-Component Gompertz Lifetime Mode, J. Statist. Comput. Simul., 2000.

[4] N. Balakrishnan, and R.S. Ambagaspitiya Relationships among moments of order statistics in samples from two related outlier models and some applications, Comm. Statist. Theory Methods, 1988.

[5] V. Barnett, and T. Lewis, Outliers in Statistical Data, Wiley, New York, 1984.

[6] L. Gavrilov, and N. Gavrilova, The biology of Life Span: A Quantitative Approach, Chur: Harwood 1991.

[7] M. Garg, B. Rao, and C. Redmond, Maximum-likelihood estimation of the parameters of the Gompertz survival function. Journal of the Royal Statistical Society, Journal of the Royal Statistical Society. Series C
TABLE V. $\quad 95 \%$ BAYESIAN PREDICTION INTERVALS FOR $y_{1}, \cdots, y_{5}$ IN THE PRESENCE OF A SINGLE OUTLIER OF TYPE $\beta+\beta_{0}$, WHERE $n=30, r=25, k=5$. NOTE: OBS. IS OBSERVATIONS PP IS POINT PREDICTORS, LB IS LOWER BOUND, UB IS UPPER BOUND, CP IS Coverage Percentages.

\begin{tabular}{|l|l|l|l|l|l|l|}
\hline$\beta_{0}$ & Obs & $y_{1}$ & $y_{2}$ & $y_{3}$ & $y_{4}$ & $y_{5}$ \\
\hline 0 & PP & 0.310392 & 0.617041 & 0.935936 & 1.29655 & 1.78929 \\
\cline { 2 - 7 } & LB & 0.009873 & 0.101746 & 0.276772 & 0.523637 & 0.873873 \\
& UB & 0.955124 & 1.35334 & 1.72163 & 2.1364 & 2.75727 \\
\cline { 2 - 7 } & Length & 0.945251 & 1.25159 & 1.44485 & 1.61277 & 1.88339 \\
\cline { 2 - 7 } & CP & $95.48 \%$ & $95.69 \%$ & $95.34 \%$ & $95.48 \%$ & $95.16 \%$ \\
\hline 1 & PP & 0.246376 & 0.518007 & 0.823625 & 1.18832 & 1.70186 \\
\cline { 2 - 7 } & LB & 0.007495 & 0.080324 & 0.227241 & 0.447124 & 0.777809 \\
& UB & 0.783238 & 1.18895 & 1.59301 & 2.04949 & 2.71016 \\
\cline { 2 - 7 } & Length & 0.775743 & 1.10863 & 1.36578 & 1.60236 & 1.93235 \\
\cline { 2 - 7 } & CP & $93.86 \%$ & $94.87 \%$ & $95.47 \%$ & $96.52 \%$ & $96.53 \%$ \\
\hline \multirow{1}{*}{2} & PP & 0.204538 & 0.469253 & 0.785086 & 1.16562 & 1.69343 \\
\cline { 2 - 7 } & LB & 0.00604 & 0.06866 & 0.203175 & 0.41544 & 0.748708 \\
& UB & 0.665036 & 1.13293 & 1.57938 & 2.04795 & 2.71014 \\
\cline { 2 - 7 } & Length & 0.658996 & 1.06427 & 1.37621 & 1.63251 & 1.96143 \\
\cline { 2 - 7 } & CP & $91.04 \%$ & $94.21 \%$ & $95.75 \%$ & $97.01 \%$ & $96.97 \%$ \\
\hline 3 & PP & 0.174974 & 0.441532 & 0.768182 & 1.15839 & 1.69166 \\
\cline { 2 - 7 } & LB & 0.005059 & 0.061072 & 0.188266 & 0.397885 & 0.736867 \\
& UB & 0.578397 & 1.11642 & 1.5783 & 2.04793 & 2.71014 \\
\cline { 2 - 7 } & Length & 0.573339 & 1.05535 & 1.39004 & 1.65004 & 1.97327 \\
\cline { 2 - 7 } & CP & $87.33 \%$ & $94.02 \%$ & $96.06 \%$ & $97.16 \%$ & $97.14 \%$ \\
\hline 4 & PP & 0.152942 & 0.424219 & 0.759568 & 1.15549 & 1.69113 \\
\cline { 2 - 7 } & LB & 0.004351 & 0.055635 & 0.17791 & 0.386966 & 0.731677 \\
& UB & 0.512017 & 1.11217 & 1.57822 & 2.04793 & 2.71014 \\
\cline { 2 - 7 } & Length & 0.507666 & 1.05654 & 1.40031 & 1.66096 & 1.97846 \\
\cline { 2 - 7 } & CP & $3.69 \%$ & $94.13 \%$ & $96.27 \%$ & $97.27 \%$ & $97.24 \%$ \\
\hline
\end{tabular}

TABLE VI. $\quad 95 \%$ BAYESIAN PREDICTION INTERVALS FOR $y_{1}, \cdots, y_{5}$ IN THE PRESENCE OF A SINGLE OUTLIER OF TYPE $\beta+\beta_{0}$, WHERE $n=30, r=30, k=0$. NOTE: OBS. IS OBSERVATIONS PP IS POINT PREDICTORS, LB IS LOWER BOUND, UB IS UPPER BOUND, CP IS Coverage Percentages.

\begin{tabular}{|l|l|l|l|l|l|l|}
\hline$\beta_{0}$ & Obs & $y_{1}$ & $y_{2}$ & $y_{3}$ & $y_{4}$ & $y_{5}$ \\
\hline \multirow{2}{*}{0} & PP & 0.309735 & 0.615638 & 0.933667 & 1.29322 & 1.78437 \\
\cline { 2 - 7 } & LB & 0.009855 & 0.10156 & 0.276229 & 0.522522 & 0.871834 \\
& UB & 0.952855 & 1.34988 & 1.71698 & 2.13035 & 2.74903 \\
\cline { 2 - 7 } & Length & 0.943 & 1.24831 & 1.44075 & 1.60783 & 1.8772 \\
\cline { 2 - 7 } & CP & $95.48 \%$ & $95.67 \%$ & $95.32 \%$ & $95.46 \%$ & $95.14 \%$ \\
\hline \multirow{1}{*}{1} & PP & 0.245765 & 0.516724 & 0.821549 & 1.18522 & 1.69719 \\
\cline { 2 - 7 } & LB & 0.007478 & 0.080147 & 0.226737 & 0.446102 & 0.77594 \\
& UB & 0.781161 & 1.18581 & 1.58871 & 2.04372 & 2.70209 \\
\cline { 2 - 7 } & Length & 0.773683 & 1.10566 & 1.36198 & 1.59762 & 1.92615 \\
\cline { 2 - 7 } & CP & $93.81 \%$ & $94.77 \%$ & $95.43 \%$ & $96.46 \%$ & $96.53 \%$ \\
\hline \multirow{1}{*}{2} & PP & 0.203983 & 0.46808 & 0.783138 & 1.16263 & 1.68881 \\
\cline { 2 - 7 } & LB & 0.006025 & 0.068498 & 0.202712 & 0.414491 & 0.74694 \\
& UB & 0.66315 & 1.13002 & 1.57519 & 2.0422 & 2.70207 \\
\cline { 2 - 7 } & Length & 0.657125 & 1.06152 & 1.37248 & 1.62771 & 1.95513 \\
\cline { 2 - 7 } & CP & $90.95 \%$ & $94.14 \%$ & $95.69 \%$ & $96.96 \%$ & $96.95 \%$ \\
\hline 3 & PP & 0.174471 & 0.44044 & 0.766305 & 1.15544 & 1.68705 \\
\cline { 2 - 7 } & LB & 0.005045 & 0.060923 & 0.187833 & 0.396987 & 0.735156 \\
& UB & 0.576681 & 1.11363 & 1.57412 & 2.04218 & 2.70207 \\
\cline { 2 - 7 } & Length & 0.571636 & 1.05271 & 1.38629 & 1.6452 & 1.96692 \\
\cline { 2 - 7 } & CP & $87.27 \%$ & $93.94 \%$ & $96.01 \%$ & $97.09 \%$ & $97.13 \%$ \\
\hline 4 & PP & 0.152484 & 0.423186 & 0.757732 & 1.15256 & 1.68652 \\
\cline { 2 - 7 } & LB & 0.004339 & 0.055497 & 0.177501 & 0.386105 & 0.729999 \\
& UB & 0.510446 & 1.10943 & 1.57404 & 2.04218 & 2.70207 \\
\cline { 2 - 7 } & Length & 0.506108 & 1.05393 & 1.39654 & 1.65608 & 1.97207 \\
\cline { 2 - 7 } & CP & $83.63 \%$ & $94.05 \%$ & $96.22 \%$ & $97.2 \%$ & $97.23 \%$ \\
\hline
\end{tabular}

(Applied Statistics), 1970.

[8] A. El-Gohary, A. Alshamrani, and A. Al-Otaibi, The generalized Gompertz distribution, Applied Mathematical Modeling, 2013.

[9] Z.F. Jaheen, Bayesian prediction under a mixture of two-component Gompertz lifetime model, Test, 2003a.

[10] Z.F. Jaheen, A Bayesian analysis of record statistics from the Gompertz model, Applied Mathematics and Computation, 2003b.

[11] A. Marshall, and I. Olkin, Life Distributions., Springer, 2007.

[12] N. Metropolis, A. W. Rosenbluth, M.N. Rosenbluth, A.H. Teller, and E. Teller, Equations of state calculations by fast computing machine, The Journal of Chemical Physics, 1953.

[13] A.F. Niazi Ali, Prediction intervals based on Gompertz doubly censored 
data, Comput Stat, 2016.

[14] C.P. Robert, and G. Casella, Moizte Ccir-io Stciristicai Methocis, New York: Springer-Verlag, 1999.

[15] A.M. Sarhan, and J. Apaloo, Exponentiated modified Weibull extension distribution, Reliability Engineering and System Safety, 2013.

[16] J. Vaupel, How change in age-specific mortality affects life expectancy, Population Studies, 1986

[17] W. Wetterstrand, Parametric models for life insurance mortality data: Gompertz's law over time, Transactions of the Society of Actuaries, 1981.

[18] F. Willekens, Gompertz in context: the Gompertz and related distributions. In Forecasting Mortality in Developed Countries - Insights from a Statistical, Demographic and Epidemiological Perspective, European Studies of Population, vol. 9, edited by E. Tabeau, A. van den Berg Jeths and C. Heathcote, Springer, 2002.
[19] W. Willemse, and H. Koppelaar, Knowledge Elicitation of Gompertz' Law of Morality, Scandinavian Actuarial Journal, 2000.

[20] M.M. El-Din, M. Nagy, and M.H. Abu-Moussa, Estimation and Prediction for Gompertz Distribution Under the Generalized Progressive Hybrid Censored Data, Annals of Data Science, 2019.

[21] M.M. Mohie El-Din, Y. Abdel-Aty, and M.H. Abu-Moussa, Statistical inference for the Gompertz distribution based on Type-II progressively hybrid censored data, Commun Stat Simul Comput, 2017.

[22] M.M. Mohie El-Din and M.H. Abu-Moussa, statistical inference and prediction for the Gompertz distribution based on multiply Type-I censored data, J Egypt Math Soc, 2018. 